\title{
On the inter ionic potentials of molten salts
}

\author{
Shigeki Matsunaga ${ }^{1}$ and Shigeru Tamaki ${ }^{2}$ \\ ${ }^{1}$ Nagaoka National College of Technology, Nagaoka 940-8532, Japan \\ ${ }^{2}$ Department of Physics, Faculty of Science, Niigata University, Niigata 950-2181, Japan
}

\begin{abstract}
Inter ionic pair potentials including the effect of ion polarization have been re-examined after the brief survey of their study. The cause of major defect of the polarizable ion model, the polarization catastrophe, has been examined. New expression of screened ion potentials including ion polarization has been proposed to avoid the catastrophe. The application to molten $\mathrm{CuI}$ has been demonstrated as an example.
\end{abstract}

\section{Introduction}

To understand qualitatively and quantitatively the properties of molten salts such as the static and dynamical behaviour, theoretical and molecular dynamics simulation studies are indispensable. For this purpose, the main problem is how to define the appropriate pairpotentials for the system of one's study.

Needless to say, the properties of molten salts are, more or less, related with those of the solid phase. There are various ionic crystals; alkali-halides with rock salt structure which has ionic bond; zinc-blend $(\mathrm{ZnS})$ type in which covalent and ionic bond coexist; superionic conductor of which a typical representative is AgI; etc.

The constant efforts have been made for about 80 years to study the inter ionic potentials for these ionic crystals of various ionisation. In this study, firstly, we will make a brief survey of the historical transition of the study of ionic potentials. Next, we will show how the theory of screened potentials is modified in connection with the recent development. The new theory will be applied to copper iodide for example. The numerical results will be discussed in comparison with those of experiment and molecular dynamics (MD) simulation.

\section{Brief survey of the study of inter ionic potentials for ionic condensed matter - Rigid lon Model -}

In 1933, Huggins and Mayer [1] proposed the inter ionic potentials effective in ionic crystals, today which are called the rigid ion model, which is expressed as,

$$
\phi_{i j}(r)=z_{i} Z_{j} e^{2} / r+B_{i j} \exp \left(-\alpha_{i j} r\right)-C_{i j} / r^{6}-D_{i j} / r^{8}
$$

where the first term in the right hand side represents the Coulomb interaction between ions $\mathrm{i}$ and $\mathrm{j}$; the second term is the repulsive potential between ions; the third term is the dipole-dipole attractive potential; the forth term is the dipole-quadrupole attractive potential. There are a number of studies on the determination of the coefficients $\mathrm{B}_{\mathrm{ij}}, \mathrm{a}_{\mathrm{ij}}, \mathrm{C}_{\mathrm{ij}}$, and $\mathrm{D}_{\mathrm{ij}}$. Famous and often used potentials of this type are known as the Tosi-Fumi potentials, which were published in 1964 [2,3]. The details of these potentials can be seen in the review articles [2-4].

Alkali halide with rock salt structure can be expressed by the central force potential (2-1). It is known that the Cauchy relation between elastic constants, $\mathrm{C}_{12}=\mathrm{C}_{44}$, is valid in ionic crystals. This potential is also effective in the study of structure or other physical properties in molten phase. Many MD studies have been reported using (2-1).

On the other hand, the Cauchy relation is not valid in many ionic crystals. The non-central force, in other words the many-body effect, has been introduced to explain the physical properties of these crystals. The effect to a certain ion from surrounding ions can be summarized as, (a) the deformation of ion shell, (b) the charge transfer between ions. We will discuss these aspects in following sections.

\section{Introduction of the potential associated with deformation of ions - Shell model -}

In the case that a cation approaches to an anion, an electron in an anion is attracted by a cation. In this case, the ionic charge number is assumed to be one for simplicity. Similarly, an electron in a cation is repulsed from an approaching cation. Schematically thinking this 
result, a cation and an anion can be treated as 'the core charge' plus 'the shell charge'. In this way, the revised potential of (2-1) can be obtained, which consists of the term of Coulomb interaction between neighbouring ions and the van der Waals force, as,

$\mathrm{U}\left(\mathrm{R}, \mathrm{W}_{1}, \mathrm{~W}_{2}\right)=\mathrm{Y}_{1} \mathrm{Y}_{2} \mathrm{e}^{2} / \mathrm{R}-\left(\mathrm{Y}_{1}-1\right) \mathrm{Y}_{2} \mathrm{e}^{2} /\left(\mathrm{R}-\mathrm{W}_{1}\right)$

$-\mathrm{Y}_{1}\left(\mathrm{Y}_{2}+1\right) \mathrm{e}^{2} /\left(\mathrm{R}+\mathrm{W}_{2}\right)+\left(\mathrm{Y}_{1}-1\right)\left(\mathrm{Y}_{2}+1\right) \mathrm{e}^{2} /\left(\mathrm{R}+\mathrm{W}_{2}-\mathrm{W}_{1}\right)$

$+\left(\mathrm{e}^{2} / 2 \mathrm{~V}\right)\left(\mathrm{k}_{1} \mathrm{~W}_{1}{ }^{2}+\mathrm{k}_{2} \mathrm{~W}_{2}{ }^{2}\right)+\mathrm{B}_{+_{-}} \exp (-\alpha \mathrm{R})-\mathrm{C}_{+-} / \mathrm{R}^{6}-\mathrm{D}_{+-} / \mathrm{R}^{8}$

The review article by Sangster and Dixon [4] is helpful to determine the shell parameters k, Y, W etc of e.g. alkali halide. There are some studies on the explanation of the mobility of ions in the super ionic conductor, however we do not go further this direction.

There is a detailed review article about the shell ion model by Cochran [5]. The following sentence can be seen in this article, which clearly expresses the shell ion model; "The model gives a satisfactory account of the polarization of ions and its coupling to the short-range interactions by considering each ion to consist of a core which is treated as a point charge and a negatively charged shell attached to its own core by a spring."

In addition, the Breathing ion model (BIM) has been proposed by Schröder [6] to explain the physical properties of ionic crystals under the assumption of the accepting deformation of ions.

In recent years, however, studies have been developed on the viewpoint of the charge distribution change in a certain ion caused by approaching of surrounding ions, rather than the ion deformation. We will discuss the former in detail in the following sections.

\section{Polarizable ion model}

In 1996, Wilson and Madden [7] succeeded to perform MD to reproduce the experimental results of molten $\mathrm{AgCl}$ structure using new potentials introducing ion polarization. In 2006, Bitrián and Trullàs [8] proposed the similar polarizable ion model to perform MD simulation in molten $\mathrm{AgBr}$. The outline of the polarizable ion model will be reviewed as follows.

In this case, different from (2-1), Vashishta-Rahman (VR) [9] type potential is adopted as the rigid ion model potential, which is familiar in the study of super ionic conductor, as,

$$
\begin{aligned}
\phi_{\mathrm{ij}}(\mathrm{r}) & =\mathrm{z}_{\mathrm{i}} \mathrm{z}_{\mathrm{j}} \mathrm{e}^{2} / \mathrm{r}+\left(\mathrm{H}_{\mathrm{ij}} / \mathrm{r}^{\mathrm{n}}\right)-\mathrm{C}_{\mathrm{ij}} / \mathrm{r}^{6}-\mathrm{P}_{\mathrm{ij}} / \mathrm{r}^{4} \\
& \equiv \phi_{\mathrm{ij}}{ }^{0}(\mathrm{r})-\mathrm{P}_{\mathrm{ij}} / \mathrm{r}^{4}
\end{aligned}
$$

where, instead of the exponential term in $(2-1), n=7$ power term is adopted for the repulsive potential in the second term on the right hand side. The dipolequadrupole interaction term is neglected, because it is smaller than the last two terms in (4-1). The feature of the polarizable ion model is that the charge-dipole interaction or the last term in (4-1), $\mathrm{P}_{\mathrm{ij}} / \mathrm{r}_{4}$, is expressed as a function of $r$, which may be the origin of its name 'polarizable'. The dipole is induced in the ion at the position $\mathbf{r}_{i}$ by the electric field $\mathbf{E}_{\mathrm{i}}$ from the neighbouring ions. The dipole moment of ion $i$ is expressed as $\mathbf{p}_{i}=\alpha_{i} \mathbf{E}_{i}$ by linear approximation, where $\alpha_{i}$ is the polarizability of ion $i$. On the contrary, the electric field from a certain ion induces the dipole moments in the surrounding ions. Therefore, $\mathbf{E}_{\mathrm{i}}$ is expressed as,

$$
\mathbf{E}_{\mathrm{i}}=\sum_{\mathrm{j} \neq \mathrm{i}}^{\mathrm{N}}\left(\mathrm{z}_{\mathrm{j}} \mathrm{e} / \mathrm{r}_{\mathrm{ij}}{ }^{3}\right) \mathbf{r}_{\mathrm{ij}}+\sum_{\mathrm{j}=\mathrm{i}}^{\mathrm{N}}\left\{3\left(\mathbf{p}_{\mathrm{j}} \cdot \mathbf{r}_{\mathrm{ij}}\right) \mathbf{r}_{\mathrm{ij}} / \mathrm{r}_{\mathrm{ij}}{ }^{3}-\left(\mathbf{p}_{\mathrm{j}} / \mathrm{r}_{\mathrm{ij}}{ }^{3}\right)\right\}
$$

where $\mathbf{r}_{\mathrm{ij}}=\mathbf{r}_{\mathrm{i}}-\mathbf{r}_{\mathrm{j}}$. The last term in (4.2) is the electric field by the dipole moment by the ion $\mathrm{j}$ around the ion $\mathrm{i}$. Needless to say, it is required that the distance between $i$ and $\mathrm{j}$ ion, $\mathrm{r}_{\mathrm{ij}}$, is much longer than the distance between the positive and negative dipole charges. In the case that the ion $\mathrm{i}$ approaches to the ion $\mathrm{j}$, the dipole becomes deformed by what is called the short range overlap effect. Then $\mathbf{p}_{\mathrm{i}}$ can be written as,

$$
\mathbf{p}_{\mathrm{i}}=\alpha_{\mathrm{i}} \mathbf{E}_{\mathrm{i}}+\alpha_{\mathrm{i}} \sum_{\mathrm{j} \neq \mathrm{i}}^{\mathrm{N}} \mathrm{s}_{\mathrm{ij}}\left(\mathrm{r}_{\mathrm{ij}}\right) \mathbf{r}_{\mathrm{ij}}
$$

Wilson and Madden [7] have set $\mathrm{s}_{\mathrm{ij}}(\mathrm{r})$ between $a$ and $b$ ions, as,

$\mathrm{S}_{\mathrm{ab}}(\mathrm{r})=-\mathrm{f}_{\mathrm{ab}}(\mathrm{r})\left(\mathrm{z}_{\mathrm{b}} \mathrm{e} / \mathrm{r}^{3}\right)$

$f_{a b}(r)$ has a value from zero to 1 depending on the inter ionic distance $r$. Putting (4-2) into (4-3), and using iteration, the dipole of the ion $a, \mathrm{p}_{\mathrm{a}}(\mathrm{r})$, is given as,

$\mathrm{p}_{\mathrm{a}}\left\{1-\left(4 \alpha_{\mathrm{a}} \alpha_{\mathrm{b}} / \mathrm{r}^{6}\right)\right\}=\alpha_{\mathrm{a}}\left\{1-\mathrm{f}_{\mathrm{ab}}(\mathrm{r})\right\}\left\{\left(\mathrm{z}_{\mathrm{b}} \mathrm{e} / \mathrm{r}^{2}\right)+\left(2 \alpha_{\mathrm{b}} \mathrm{z}_{\mathrm{a}} \mathrm{e} / \mathrm{r}^{5}\right)\right\}$

or

$\mathrm{p}_{\mathrm{a}}=\alpha_{\mathrm{a}}\left\{1-\mathrm{f}_{\mathrm{ab}}(\mathrm{r})\right\}\left\{\left(\mathrm{z}_{\mathrm{b}} \mathrm{e} / \mathrm{r}^{2}\right)+\left(2 \alpha_{\mathrm{b}} \mathrm{z}_{\mathrm{a}} \mathrm{e} / \mathrm{r}^{5}\right)\right\} /\left\{1-\left(4 \alpha_{\mathrm{a}} \alpha_{\mathrm{b}} / \mathrm{r}^{6}\right)\right\}$

which means $p_{a}(r)$ diverges at $r_{c}{ }^{6}=\left(4 \alpha_{a} \alpha_{b}\right)$. This singular point is called the polarization catastrophe distance. This divergence is ascribed to the fact that the iteration has been done disregarding that $r_{i j}$ is required much longer than the distance between the positive and negative dipole charges. In the case that $\mathrm{a}=\mathrm{b},(4-6)$ is,

$\mathrm{p}_{\mathrm{a}}=\alpha_{\mathrm{a}}\left\{1-\mathrm{f}_{\mathrm{ab}}(\mathrm{r})\right\}\left\{\mathrm{z}_{\mathrm{a}} \mathrm{er} /\left(\mathrm{r}^{3}-2 \alpha_{\mathrm{a}}\right)\right\}$

which means the divergence occurs at $r^{3}=2 \alpha_{a}$. The dipole-dipole interaction potentials on the basis of the polarizable ion model can be obtained from (4-6) and (4$7)$ as follows. Suppose that there are two ions $a$ and $b$. The charge dipole interaction potentials at the position of ions $a$ and $b$ are $\mathrm{p}_{\mathrm{a}} \mathrm{E}_{\mathrm{a}}$ and $\mathrm{p}_{\mathrm{b}} \mathrm{E}_{\mathrm{b}}$, respectively. Therefore,

$\mathrm{P}_{\mathrm{ij}} / \mathrm{r}^{4}=(1 / 2)\left(\mathrm{p}_{\mathrm{a}} \mathrm{E}_{\mathrm{a}}+\mathrm{p}_{\mathrm{b}} \mathrm{E}_{\mathrm{b}}\right)$

The contribution from $\mathrm{p}_{\mathrm{a}} \mathrm{p}_{\mathrm{b}}$ in the right hand side, i.e. the dipole-dipole interaction, should have already been taken into account in $\mathrm{C}_{\mathrm{ij}} / \mathrm{r}^{6}$. 


$$
\begin{aligned}
& -\mathrm{P}_{\mathrm{ij}} / \mathrm{r}^{4}=-(1 / 2)\left(\mathrm{p}_{\mathrm{a}} \mathrm{E}_{\mathrm{a}}+\mathrm{p}_{\mathrm{b}} \mathrm{E}_{\mathrm{b}}\right)=\phi_{\text {charge-dipole }}(\mathrm{r}) \\
& =-(1 / 2)\left\{1-\mathrm{f}_{\mathrm{ab}}(\mathrm{r})\right\}^{2}\left\{\left(\alpha_{\mathrm{a}} \mathrm{z}_{\mathrm{b}} \mathrm{e}^{2}+\alpha_{\mathrm{b}} \mathrm{z}_{\mathrm{a}} \mathrm{e}^{2}\right) \mathrm{r}^{2}+\mathrm{z}_{\mathrm{a}} \mathrm{z}_{\mathrm{b}} \mathrm{e}^{2} \mathrm{r}_{\mathrm{c}}{ }^{6} / \mathrm{r}\right\} /\left(\mathrm{r}^{6}-\mathrm{r}_{\mathrm{c}}{ }^{6}\right)
\end{aligned}
$$

Then, we have,

$$
\begin{aligned}
\phi_{+-}(\mathrm{r})=\phi_{+-}^{0}(\mathrm{r}) & -(1 / 2)\left\{1-\mathrm{f}_{++}(\mathrm{r})\right\}^{2}\left\{\left(\alpha_{+} \mathrm{z}^{-} \mathrm{e}^{2}+\alpha_{-} \mathrm{z}^{+} \mathrm{e}^{2}\right) \mathrm{r}^{3}\right. \\
+ & \left.\mathrm{z}^{+} \mathrm{z}^{-} \mathrm{e}^{2} \mathrm{r}_{\mathrm{c}}{ }^{6}\right\} / \mathrm{r}\left(\mathrm{r}^{6}-\mathrm{r}_{\mathrm{c}}{ }^{6}\right)
\end{aligned}
$$

$\phi_{++}(\mathrm{r})=\phi_{++}{ }^{0}(\mathrm{r})-\left\{1-\mathrm{f}_{++}(\mathrm{r})\right\}^{2}\left\{\left(\alpha_{+} \mathrm{z}^{+} \mathrm{e}^{2} \mathrm{r}^{3}+4 \mathrm{z}^{+2} \mathrm{e}^{2} \alpha_{+}{ }^{2}\right) / \mathrm{r}\left(\mathrm{r}^{6}-4 \alpha_{+}{ }^{2}\right)\right.$

$\phi_{--}(\mathrm{r})=\phi_{--}{ }^{0}(\mathrm{r})-\left\{1-\mathrm{f}_{-}(\mathrm{r})\right\}^{2}\left\{\left(\alpha_{-} \mathrm{z}^{-} \mathrm{e}^{2} \mathrm{r}^{3}+4 \mathrm{z}^{-2} \mathrm{e}^{2} \alpha_{-}^{2}\right) / \mathrm{r}\left(\mathrm{r}^{6}-4 \alpha_{-}{ }^{2}\right)\right.$

Therefore, strictly speaking, these equations are valid only in the case when the polarizability of the ion $a$ or $b$ is almost zero. In the molten state of $\mathrm{Ag}$ or $\mathrm{Cu}$ halide including super ionic conductor, e.g. $\mathrm{AgI}, \mathrm{AgBr}, \mathrm{Ag}_{2} \mathrm{Se}$, $\mathrm{Ag}_{2} \mathrm{Te}, \mathrm{CuBr}$ etc., the dipole moments of cations are considerably smaller than those of anions, hence $r_{c}{ }^{6} \sim 0$ [10]. Consequently, the condition $\alpha_{+} \sim 0$ would be strictly applicable to $\phi_{+-}(\mathrm{r})$ and $\phi_{++}(\mathrm{r})$. Moreover, approximating the denominator in (4-12) as $r^{7}$, then all potentials can be set up. From the considerations based on these facts, the polarizable ion model has been applied to $\mathrm{AgCl}$ and $\mathrm{AgBr}[7,8]$.

\section{Evaluation of Polarizable Ion Model in the previous section}

The theory that Bitrián and Trullàs [8] have developed in the previous section is effective to revise the rigid ion model to some extent. However, as we have pointed out in the previous section, such dispersion called the polarization catastrophe is ascribed to the fact that the iteration has been done disregarding that the inter ionic distance $r_{i j}$ is required to be much longer than the distance between the positive and negative dipole charges. In fact, for example, the polarization catastrophe distance in molten $\mathrm{NaCl}$ estimated from the electron polarizabilities of $\mathrm{Na}$ and $\mathrm{Cl}$ ions is $0.93 \mathrm{~A}$. Of course the actual negative increasing of potential to the catastrophe distance would be relaxed to some extent, as the short range damping factor $f_{a b}(r)$ rapidly approaches to 1 . In spite of these facts, it is quite doubtful whether these are effective theoretical value when $r$ approaches to the nearest ionic distance.

Because the $\mathrm{f}_{\mathrm{ab}}(\mathrm{r})$ is used as a parameter, the following a prior expression seems to be more preferable, as,

$\phi_{\mathrm{ab}}(\mathrm{r})=\phi_{\mathrm{ab}}{ }^{0}(\mathrm{r})-\mathrm{P}_{\mathrm{ab}} / \mathrm{r}^{4}$
$=\phi_{\mathrm{ab}}{ }^{0}(\mathrm{r})-(1 / 2)\left\{1-\mathrm{f}_{\mathrm{ab}}(\mathrm{r})\right\}^{2}\left(\alpha_{\mathrm{a}} \mathrm{z}_{\mathrm{b}}{ }^{2} \mathrm{e}^{2}+\alpha_{\mathrm{b}} \mathrm{z}_{\mathrm{a}} \mathrm{e}^{2}\right) / \mathrm{r}^{4}$

Though $f_{a b}(r)$ in (5-1) is different from those Wilson and Madden [7], or Bitrián and Trullàs [8] have defined, the function which satisfies following condition will be appropriate; when $r$ approaches to 0 , then $\mathrm{f}_{\mathrm{ab}}(\mathrm{r})$ approaches to 1; when $r$ increases (about twice of the inter ionic distance), $\mathrm{f}_{\mathrm{ab}}(\mathrm{r})$ approaches to 0 . In any case, by setting $f_{a b}(r)$ as $(5-1)$, the repulsive part of the potential will be more relaxed by taking into account the last term in $(5-1)$, the charge dipole interaction, in the range where the repulsive potential is effective.

\section{Polarization and dielectric constant}

To discuss the screened inter ionic potential by the dielectric function in the next section, we will briefly summarize the relation between the polarization and the dielectric constant. Suppose that the local electric field at the position of the ion is $\mathrm{E}_{\mathrm{loc}}$, the contribution to the polarizability is ordinary classified to the following three types; (a) the electron distribution change of the atomic core which occurs inside the ion. It has been discussed in the previous section; (b) the contribution from ions, which occurs from the relative configuration of other neighbouring ions; (c) if the ion has the permanent electric dipole, its contribution should be considered, specially in the case that the ion consists of plural atoms. However, it is unnecessary to be taken into account in molten alkali chloride or molten carbonate.

In the solid phase, if the local electric field $E_{\text {loc }}$ is applied to the frequency analysis, $c$ vanishes at about the micro wave length; next $b$ disappears at the infrared region; $a$ lasts to the end. In this discussion, we pay attention to $a$ and $b$.

According to the linear response theory, the relation between the electron polarizability discussed in the previous sections and the dielectric constant corresponding to it is expressed as,

$$
1-(1 / \varepsilon(q))=4 \pi \alpha(q)
$$

Therefore, it is possible to obtain the effective inter ionic potentials in molten salt by calculation the term $a$ and $b$ using the polarization or the dielectric constant.

\section{Our final form of inter- ionic potentials in molten salts}

Koishi, Saito, Matsunaga and Tamaki [11-13] have derived the dielectric function of the attractive part of the inter ionic potential of molten salts. We briefly describe the procedure as follows. The Fourier component of the Coulomb potential in $\phi_{\mathrm{ij}}{ }^{0}(\mathrm{r})$, i.e. $\mathrm{FT}\left[\mathrm{z}_{\mathrm{i}} \mathrm{z}_{\mathrm{j}}{ }^{2} / \mathrm{r}\right]=4 \pi \mathrm{z}_{\mathrm{i}} \mathrm{z}_{\mathrm{j}} \mathrm{e}^{2} / \mathrm{q}^{2}$ is screened by the dielectric function, as,

$\left\{\right.$ the result of the screened Coulomb term in $\left.\phi_{\mathrm{ij}}^{0}(\mathrm{r})\right\}$
\[ =4 \pi \mathrm{z}_{\mathrm{i}} \mathrm{z}_{\mathrm{j}}{ }^{2} / \mathrm{q}^{2} \varepsilon(\mathrm{q}) \]

As will be described in the next section, $\varepsilon(\mathrm{q})$ is related to the charge-charge structure factor $\operatorname{Szz}(q)$. In other words, $\varepsilon(q)$ is introduced to the pair potential as, so to say, a factor of the many body effect, or the environmental factor. Adding the repulsive term $\left(\zeta \mathrm{H}_{\mathrm{ij}} / \mathrm{r}^{\mathrm{n}}\right)$ and the dipoledipole interaction term $\left(-\xi \mathrm{C}_{\mathrm{ij}} / \mathrm{r}^{6}\right)$ in $\phi_{\mathrm{ij}}{ }^{0}(\mathrm{r})$ with the simplified reduction constants $\zeta$ and $\xi$ to (7-1), the promise inter-ionic potentials in $a-b$ binary molten salt screened by the many-body force are expressed as, 


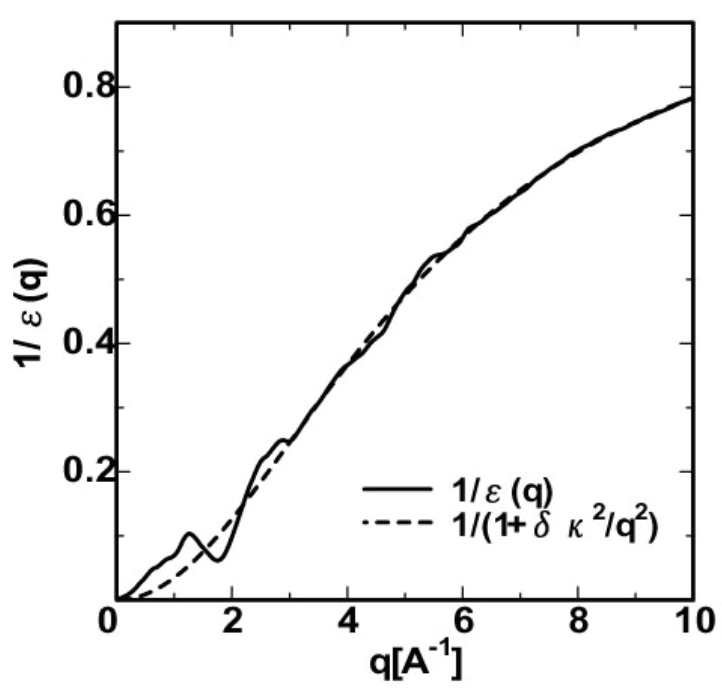

Fig. 1. $1 / \varepsilon(\mathrm{q})$ of molten $\mathrm{CuI}$ with $1 /\left(1+\delta\left(\kappa_{\mathrm{s}}{ }^{2} / \mathrm{q}^{2}\right)\right)$.

$$
\begin{aligned}
& \phi_{\mathrm{ij}}(\mathrm{r}) \equiv \operatorname{Inv} \cdot \mathrm{FT}\left[4 \pi \mathrm{z}_{\mathrm{i}} \mathrm{z}_{\mathrm{j}} \mathrm{e}^{2} / \mathrm{q}^{2} \varepsilon(\mathrm{q})\right]+\zeta \mathrm{H}_{\mathrm{ij}} / \mathrm{r}^{\mathrm{n}} \\
& \quad-(1 / 2)\left\{1-\mathrm{f}_{\mathrm{ij}}(\mathrm{r})\right\}^{2}\left(\alpha_{\mathrm{i}} \mathrm{z}_{\mathrm{j}} \mathrm{e}^{2}+\alpha_{\mathrm{j}} \mathrm{z}_{\mathrm{i}} \mathrm{e}^{2}\right) / \mathrm{r}^{4}-\xi \mathrm{C}_{\mathrm{ij}} / \mathrm{r}^{6} \quad(\mathrm{i}, \mathrm{j}=\mathrm{a}, \mathrm{b})
\end{aligned}
$$

where Inv.FT means the inverse Fourier transformation. The repulsive term in (7-2) can be replaced by the exponentially decaying functions.

Provided that the following function (7-3) is adopted as the decaying factor $f_{a b}(r)$, the desirable condition will be satisfied; as $r$ approaches to 0 , then $\mathrm{f}_{\mathrm{ab}}(\mathrm{r})$ approaches to 1 ; as $r$ increases, then $f_{a b}(r)$ approaches to 0 .

$\mathrm{f}_{\mathrm{ab}}(\mathrm{r})=2 /\left\{\exp \left(+\mathrm{k}_{\mathrm{ab}} \mathrm{r}\right)+\exp \left(-\mathrm{k}_{\mathrm{ab}} \mathrm{r}\right)\right\}$

where $\mathrm{k}_{\mathrm{ab}}$ is a variable parameter, e.g. if $\mathrm{f}_{+-}(\mathrm{r}) \sim 0.2$ is required when the inter ionic distance is about $3 \mathrm{~A}$, the estimated value is $\mathrm{k}_{+-} \sim 0.77 \mathrm{~A}^{-1}$. Regarding $\varepsilon(\mathrm{q})$, the detailed discussion can been found in our previous works [11-13]. As we have stated so far, the polarizable ion model in the short-range distance has been adopted in (72) where the repulsive potential (in both case $1 / \mathrm{r}^{\mathrm{n}}$ function form and the exponentially decaying function) is dominant. On the other hand, the screening contribution is adopted in the long-range distance where the Coulomb attractive potential is dominant. Thus the plausible inter ionic potential can be obtained, as it were, the polarized and screened inter-ionic potentials model (PSIPM). We will demonstrate the example in the next section.

\section{Example of application}

In this section, we will show the example of application of the new theory to molten CuI. In molten $\mathrm{CuI}$, the anomaly approach in $\mathrm{Cu}-\mathrm{Cu}$ distribution is known, which is not reproduced by the classical MD [14]. Firstly, the outline of the theory of the dielectric screening that we have developed in the previous work will be briefly described as follows for the readers' benefit [11-13]. As stated in the previous section, the Coulomb potential is screened by the dielectric constant $1 / \varepsilon(\mathrm{q})$. $\quad \varepsilon(\mathrm{q})$ is expressed using the charge-charge structure factor, $\mathrm{S}_{\mathrm{ZZ}}(\mathrm{q})$, which is defined as [15],

$S_{Z Z}(q)=<\rho_{q}{ }^{Z} \rho_{-q}^{Z}>/ N=\Sigma_{i} \Sigma_{j} Z_{i} Z_{j} S_{i j}(q)$

where $z_{i}$ and $z_{j}$ are the effective charge of ions, $S_{i j}(q)$ is the partial structure factor, i.e. the Fourier transformation of the pair distribution function of ion $\mathrm{i}$ and $\mathrm{j}, \mathrm{g}_{\mathrm{ij}}(\mathrm{r})$, as,

$\mathrm{S}_{\mathrm{ij}}(\mathrm{q})=\mathrm{x}_{\mathrm{i}} \delta_{\mathrm{ij}}+\mathrm{nx}_{\mathrm{i}} \mathrm{x}_{\mathrm{j}} \int_{0}^{\infty}(\sin \mathrm{qr} / \mathrm{qr})\left\{\mathrm{g}_{\mathrm{ij}}(\mathrm{r})-1\right\} 4 \pi \mathrm{r}^{2} \mathrm{dr}$

$\mathrm{S}_{\mathrm{ij}}(\mathrm{q})$ and $\mathrm{g}_{\mathrm{ij}}(\mathrm{r})$ directly reflect the ionic configuration and are easily obtainable from neutron diffraction experiment or molecular dynamics simulation.

In the case that an external charge $\mathrm{e} \rho_{\mathrm{ext}}(\mathbf{r})$ is introduced from outside into this system at the position $\mathbf{r}$, then a charge $e \rho_{\text {ind }}(\mathbf{r})$ is induced. By the Gauss's law, the divergence of the electric displacement $\boldsymbol{D}(\mathbf{r})$ and the electric field $\boldsymbol{E}(\mathbf{r})$ are related to $\mathrm{e} \rho_{\mathrm{ext}}(\mathbf{r})$ and $\mathrm{e} \rho_{\text {ind }}(\mathbf{r})$, respectively. The screened charge is expressed as $\rho_{\text {ext }}(\mathbf{r}) / \varepsilon$. In general, the dielectric constant $\varepsilon$ may be extended to an isotropic r-dependent term written as $\varepsilon(\mathrm{r})$. Besides, $\rho_{\text {ext }}(\mathbf{r}) / \varepsilon(r)$ can be replaced by $\rho_{\text {ext }}(\mathbf{r})+\rho_{\text {ind }}(\mathbf{r})$. Taking the ratio of the Fourier transformation of the Gauss's law for e $\rho_{\text {ind }}(\mathbf{r})$ to e $\rho_{\text {ext }}(\mathbf{r})$, the inverse dielectric function $1 / \varepsilon(q)$ is expressed as follows,

$1 / \varepsilon(q)=1+\left\{\rho_{\text {ind }}(\mathbf{q}) / \rho_{\text {ext }}(\mathbf{q})\right\}$

where we assume $\varepsilon(q)$ is isotropic, for simplicity.

Meanwhile, the electric potential $\phi(\mathbf{r})$ due to this external charge density is defined as,

$\boldsymbol{E}(\mathbf{r})=-\operatorname{grad} \phi(\mathbf{r})$

Using the Gauss's law, we have the Poisson equation, as,

$\nabla^{2} \phi(\mathrm{r})=-\left\{4 \pi \mathrm{e} \rho_{\mathrm{ext}}(\mathrm{r}) / \varepsilon(\mathrm{r})\right\}$

Putting the Fourier representations of $\varepsilon(\mathrm{r}), \phi(\mathbf{r}), \rho_{\mathrm{ext}}(\mathbf{r})$ and $\rho_{\text {ind }}(\mathbf{r})$, into eq.(8-5) and taking $\mathbf{r}=0$, then we have,

$\mathrm{q}^{2} \varepsilon(\mathrm{q}) \phi(\mathbf{q})=4 \pi e \underline{\rho}_{\mathrm{ext}}(\mathbf{q})$

e $\varrho_{\text {ind }}(\mathbf{r})$ is the charge fluctuation yielded by the insertion of the external charge e $\rho_{\text {ext }}(\mathbf{r})$, where the under bar stands for vector. Therefore $e_{\text {ind }}(\mathbf{r})$ might be expressed in the following form,

$e \rho_{\text {ind }}(\mathbf{q})=\chi_{z z}(q) e \phi_{\text {ext }}(\mathbf{q})$

where $\chi_{z z}(q)$ is the charge response function. On the basis of the fluctuation dissipation theorem, $\chi_{z z}(q)$ is expressed in terms of $\mathrm{S}_{\mathrm{ZZ}}(\mathrm{q})$ as [15],

$\chi_{z Z}(q)=-\beta n S_{Z Z}(q)$ 


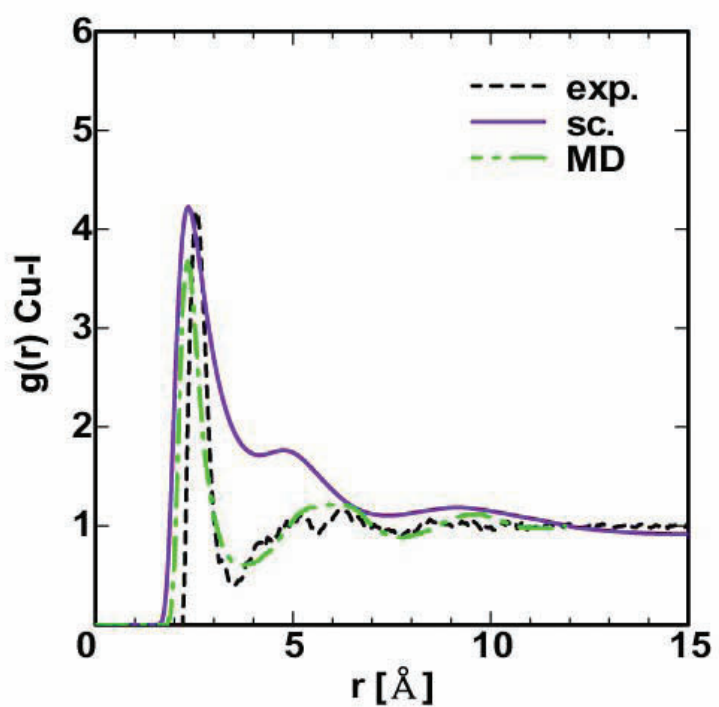

Fig. 2. $\mathrm{g}_{\mathrm{CuI}}$ at $940 \mathrm{~K}$ calculated using the screened potential (solid line) with that obtained by experiment (broken line) and by MD (broken dotted line).

Putting (8-6) and (8-7) into (8-3), and using (8-8), we have the relation between $1 / \varepsilon(\mathrm{q})$ and $\mathrm{S}_{\mathrm{ZZ}}(\mathrm{q})$, as,

$$
\frac{1}{\varepsilon(\mathrm{q})}=\frac{1}{1+\delta\left(\kappa^{2} / \mathrm{q}^{2}\right) \mathrm{S}_{\mathrm{ZZ}}(\mathrm{q})}
$$

where $\kappa_{\mathrm{s}}^{2}=4 \pi \mathrm{e}^{2} \beta \mathrm{n}, \beta=1 / \mathrm{k}_{\mathrm{B}} \mathrm{T}$, and $\mathrm{n}$ is the number density of constituent ions. The parameter $\delta$ is introduced to reduce the screening effect by the structure anisotropy as seen in molten noble metal halide. The value of $\delta$ is supposed to be in the range of $0<\delta \leq 1$. The condition $\delta=$ 1 may occur in a well-symmetric configuration like molten alkali halide. In the region of high values of $q>$ $10 \mathrm{~A}^{-1}, \mathrm{~S}_{\mathrm{zZ}}(\mathrm{q}) \cong 1$ is valid, hence (8-9) will be written as,

$$
\frac{1}{\varepsilon_{\mathrm{i}}(\mathrm{q})}=\frac{1}{1+\delta\left(\kappa^{2} / \mathrm{q}^{2}\right)}
$$

As we have describes in the previous section, the screened potentials can be obtained using $\varepsilon(q)$. In our previous work, to confirm the validity of the obtained potential, we have calculated the pair distribution function by the potential mean force [15], which is expressed as,

$g_{\mathrm{ij}}(r)=\exp \left[-U^{\mathrm{ij}}(\mathrm{r}) / \mathrm{k}_{\mathrm{B}} \mathrm{T}\right]$

In our previous work, however, the screened potential $\phi_{\mathrm{ij}}(\mathrm{r})$ with the small reduction parameters multiplied on the repulsive term was used for $\mathrm{U}^{\mathrm{ij}}(\mathrm{r})$, and the calculation was restricted to the cation and anion pair.

On this stage, we try to make a slight modification of the interpretation of the theory. We consider two types of screened potentials; the first one, $\phi_{\mathrm{ij}}{ }^{\mathrm{S}}(\mathrm{r})$, is the screened potential that we have discussed in the previous section,

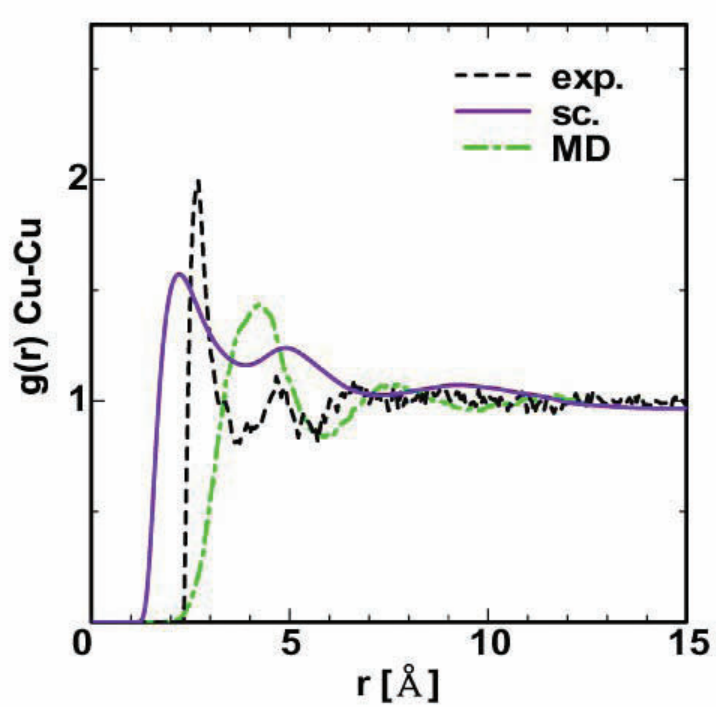

Fig. 3. $\mathrm{g}_{\mathrm{CuCu}}$ at $940 \mathrm{~K}$ calculated using the screened potential (solid line) with that obtained by experiment (broken line) and by MD (broken dotted line)..

which is affected by the structure through $\mathrm{S}_{\mathrm{ZZ}}(\mathrm{q})$; the second one, $\phi_{\mathrm{ij}}^{\mathrm{I}}(\mathrm{r})$, is so to say, the 'ideal' screened potential with $\varepsilon_{i}(q)$ in $(8-10)$, which expresses the effect from the uniform back ground. The potentials are written as,

$$
\begin{aligned}
& \phi_{\mathrm{ij}}^{\mathrm{S}}(\mathrm{r}) \equiv \operatorname{Inv} \cdot \mathrm{FT}\left[4 \pi \mathrm{z}_{\mathrm{i}} \mathrm{z}_{\mathrm{j}} \mathrm{e}^{2} / \mathrm{q}^{2} \varepsilon(\mathrm{q})\right]+\zeta^{\mathrm{S}} \mathrm{H}_{\mathrm{ij}} / \mathrm{r}^{\mathrm{n}} \\
& -(1 / 2)\left\{1-\mathrm{f}_{\mathrm{ij}}(\mathrm{r})\right\}^{2}\left(\alpha_{\mathrm{i}} \mathrm{z}_{\mathrm{j}} \mathrm{e}^{2}+\alpha_{\mathrm{j}} \mathrm{z}_{\mathrm{i}} \mathrm{e}^{2}\right) / \mathrm{r}^{4}-\xi^{\mathrm{S}} \mathrm{C}_{\mathrm{ij}} / r^{6} \\
& \phi_{\mathrm{ij}}^{\mathrm{I}}(\mathrm{r}) \equiv \operatorname{Inv} \cdot \mathrm{FT}\left[4 \pi \mathrm{z}_{\mathrm{i}} \mathrm{z}_{\mathrm{j}} \mathrm{e}^{2} / \mathrm{q}^{2} \varepsilon_{\mathrm{i}}(\mathrm{q})\right]+\zeta^{\mathrm{I}} \mathrm{H}_{\mathrm{ij}} / \mathrm{r}^{\mathrm{n}} \\
& -(1 / 2)\left(\alpha_{\mathrm{i}} \mathrm{z}_{\mathrm{j}} \mathrm{e}^{2}+\alpha_{\mathrm{j}} \mathrm{z}_{\mathrm{i}} \mathrm{e}^{2}\right) / \mathrm{r}^{4}-\xi^{\mathrm{I}} \mathrm{C}_{\mathrm{ij}} / \mathrm{r}^{6}
\end{aligned}
$$$$
\text { - (1/2)\{1- } \left.\mathrm{f}_{\mathrm{ij}}(\mathrm{r})\right\}^{2}\left(\alpha_{\mathrm{i}} \mathrm{z}_{\mathrm{j}} \mathrm{e}^{2}+\alpha_{\mathrm{j}} \mathrm{z}_{\mathrm{i}} \mathrm{e}^{2}\right) / \mathrm{r}^{4}-\xi^{\mathrm{S}} \mathrm{C}_{\mathrm{ij}} / \mathrm{r}^{6} \quad(\mathrm{i}, \mathrm{j}=\mathrm{a}, \mathrm{b})
$$

We take $\mathrm{U}^{\mathrm{ij}}(\mathrm{r})$ for the potential of mean force in (8-11) as the deviation from the ideal screening, as,

$$
\mathrm{U}^{\mathrm{ij}}(\mathrm{r}) \equiv-\Delta \phi_{\mathrm{ij}}(\mathrm{r})=-\left|\phi_{\mathrm{ij}}^{\mathrm{S}}(\mathrm{r})-\phi_{\mathrm{ij}}^{\mathrm{I}}(\mathrm{r})\right|
$$

This interpretation seems to be appropriate because the effective mean force is originally defined for the weak interaction between particles, which corresponds to $\Delta \phi_{\mathrm{ij}}(\mathrm{r})$ in this case. In the numerical calculation, we adopt the value of $\delta$ in (8-9) and (8-10) as $0.4, \Delta \zeta=\mid \zeta^{\mathrm{S}}$. $\zeta^{\mathrm{I}} \mid=0.12 . \mathrm{C}_{\mathrm{CuCu}}$ and $\mathrm{C}_{\mathrm{CuI}}$ are 0 in (8-12) and (8-13).

The obtained $g_{+}(r)$ and $g_{++}(r)$ are shown in figure 2 and figure 3 with $\mathrm{g}_{\mathrm{ij}}(\mathrm{r})$ obtained by experiment [14] and MD using the original VR type potentials [16]. The first peak of $g_{+-}(r)$ and the phase of oscillations almost agree well, though the second peak height is not satisfactory reproduced. The first peak position of $g_{++}(r)$ obtained by the experiment suggests the anomalous closer distribution of $\mathrm{Cu}$ ions, which has been reproduced by the screened potentials and the effective mean force to some extent, except their peak heights and the oscillation phase of the third peak. On the other hand, the first peak of $g_{++}(r)$ obtained by MD obviously disagrees with the experiment. 
The discrepancy between $g_{\mathrm{ij}}(\mathrm{r})$ by the experiment and that obtained by the screened potential may be caused by several reasons, in addition to a margin of error in the Fourier transformation. One of the expected reasons is that the non-symmetric structure in solid phase remains in molten phase, which may obstruct the screening effect in molten phase. Despite these facts, however, it may be suggested that the treatment described so far is physically significant, and the screening effect has the obvious contribution to the obtained results.

\section{Conclusion}

The theory of dielectric screening in molten salt has been applied to the inter ionic potential with the polarization. The new expression of the polarizable and screened interionic potential model has been proposed considering the cause of the polarization catastrophe. The example of application of the theory has been demonstrated using the potential of mean force in molten $\mathrm{CuI}$ with $\mathrm{MD}$ result. The obtained pair distribution functions have reproduced the feature of the experimental results to some extent.

\section{Acknowledgements}

One of the authors (SM) is grateful to the Ministry of Education, Science and Culture for Financial support of Grant-in-Aid for Science Research. One of authors (ST) expresses his thanks to Professors S. Takeno and M. Kusakabe of Niigata Institute of Technology for their helpful instruction for a mathematical treatment. He also wishes to express his cordial thanks to Professor W. H. Young for fruitful comments on this subject.

\section{References}

[1] M. L. Huggins and J. E. Mayer, J. Chem. Phys., 1, 643 (1933)

[2] M. P. Tosi, , Solid State Physics, 16, 1. (Academic

Press, 1964)

[3] M. P. Tosi, and F. G. Fumi, J. Phys. Chem. Solids, 25, 45 (1964)

[4] M. J. L. Sangster and M.Dixon, Advances in Physics, 25, 247 (1976)

[5] W.Cochran, CRC Critical Rev. in Solid St. Sciences, 2, 1. (1971)

[6] U. Schröder, Solid State Commun., 4, 347 (1966)

[7] M. Wilson, P. A. Madden and B. J. Costa-Cabral, J. Phys. Chem. 100, 1227 (1996)

[8] V. Bitrián and J. Trullàs, J. Phys. Chem. 110, 7490

(2006)

[9] P. Vashishta and A. Rahman, Phys. Rev. Lett. 40,

1337, (1978)

[10] see, e.g., the table of polarizability of ions in C.

Kittel, Introduction to Solid State Physics 7th ed(Wiley, New York, 1996)

[11] T. Koishi, M. Saito, S. Matsunaga and S. Tamaki, Phys. Chem. Liq. 45, 181 (2007)

[12] S. Matsunaga, M. Saito, T. Koishi and S. Tamaki, Mol. Sim. 33, 153 (2007)
[13] S. Matsunaga, M. Saito, T. Koishi and S. Tamaki, J. Alloys Compounds, 452, 182 (2008)

[14] Y. Waseda, S. Kang, K. Sugiyama, M. Kimura and M. Saito, J. Phys.: Condens. Matter 12, A195 (2002)

[15] J.P. Hansen and I.R. McDonald. Theory of Simple Liquids $2^{\text {nd }}$ ed, (Academic Press, New York, 1986)

[16] A. J. Stafford, M. Silbert, J. Trullàs and A. Diró, J. Phys.: Condens. Matter 2, 6631 (1990) 